\title{
Handwritten Digit Recognition using various Neural Network Approaches
}

\author{
Sakshica ${ }^{1}$, Dr. Kusum Gupta ${ }^{2}$ \\ Banasthali Vidyapith, Jaipur Campus, Jaipur ${ }^{1,2}$
}

\begin{abstract}
Handwritten digit recognition is one of the important problems in computer vision these days. There is a great interest in this field because of many potential applications, most importantly where large number of documents must be dealed such as post mail sorting, bank cheque analysis, handwritten form processing etc. So a system should be designed in such a way that it is capable of reading handwritten digits and provide appropriate results. This paper presents a survey on various neural network approaches to recognize handwritten digits.
\end{abstract}

Keywords: Artificial Neural Network (ANN), Handwritten Digit Recognition, Back-propagation (BP), Single Layer Perceptron (SLP), Hopfield Neural Network (HNN).

\section{INTRODUCTION}

Human beings have been given a natural tendency to identify letters, objects, numbers, and voices. However, making a machine do these types of tasks is a very complicated problem. Pattern recognition is a necessary component of artificial intelligence and computer vision. Interest in pattern recognition is growing fast aligned to the prohibitive amount of information that we come across in our day-to-day. Consequently, computerization is very much needed to handle this information explosion [1].

Handwritten digits recognition is an old and important problem of machine learning. The objective is to recognize images of isolated handwritten digits (0-9). More specifically, the problem is equivalent to find a model, which takes image of handwritten digit as input, and output the predicted class label of the image [2]. Furthermore, the ideas and methods to solve this problem would be very useful in various fields of pattern recognition problems where large volumes of real-world data is used [1].

Neural network has been widely used in the field of pattern recognition. This paper presents survey on handwritten digit recognition using various neural network approaches such as Single layer perceptron (SLP) model, Back-propagation (BP) algorithm and Hopfield Neural Network (HNN) model.

\section{Artificial NeUral Network}

An Artificial Neural Network (ANN) is an information processing model which is inspired by the biological nervous systems, such as our brain. It is composed of a large number of highly interconnected processing elements called neurons working in coordination to solve specific problems [8].

A Neural Network is designed for the process of pattern recognition or data classification, through a learning process. In biological systems, Learning involves adjustments to the synaptic connections existing between neurons called weights. Neural networks process information in the same way as the human brain does. The network is composed of a large number of highly interconnected processing elements working in parallel to solve a specific problem. Neural networks learn by example [8].

In this paper the section III gives the brief introduction of the various stages of handwritten digit recognition. Section IV gives a brief introduction of various artificial neural network techniques used for the classification of handwritten digits. Section V gives the conclusion and future scope of the described work.

\section{III.VARIOUS STAGES OF RECOGNITION}

The Handwritten digit recognition system has to go through following step [3]:

Image acquisition

Preprocessing

Segmentation

Feature extraction

Classification

Image acquisition implies obtaining the image either by scanning documents or by capturing photograph or by directly writing using mouse [3].

Pre-processing of the image means applying a number of procedures for the purpose of thresholding, smoothing, filtering, resizing, and normalizing so that further algorithm for final classification can be made simple and more accurate [4].

Image segmentation means to divide the image into various segments so that it can be made more meaningful and easy to analyze [3].

Feature Extraction is the process to capture appropriate characteristics of the target object. It is mainly related to dimension reduction. The extracted features from an image are applied as an input to the train classifier like neural network [3].

After obtaining feature vector from the input image next step is to decide which classifier to use for classifying the class of handwritten digits. The most traditional classifier to be used is neural network [3]. 


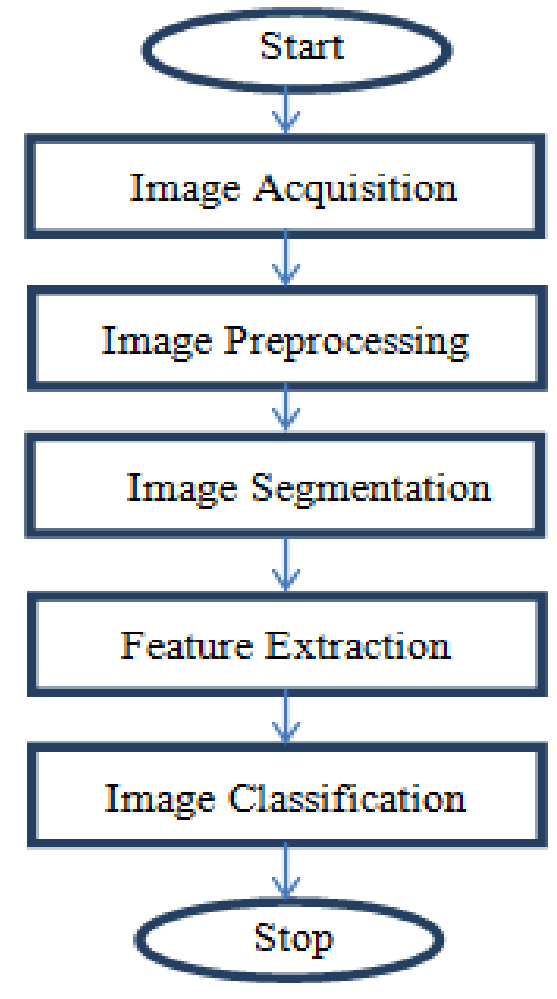

Fig 1: Flow chart for digit recognition system [3]

\section{IV.CLASSIFICATION USING VARIOUS NEURAL NETWORK APPROACHES}

Classification determines the region or area of feature space in which an unknown pattern falls. The various neural network approaches used for classification are described as follows [6]:

\section{A. Single layer perceptron model}

The basic structure of a single layer perceptron is described with the help of Fig.2. We can consider it as a single "neuron" which has various input signals that provide an output signal [6].

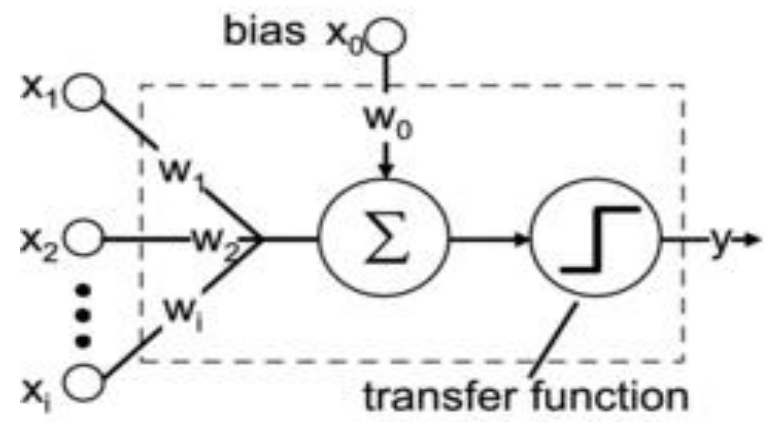

Fig.2: Single layer perceptron network

$$
\mathrm{Xj}=\sum_{i=1}^{k} w_{\mathrm{ij}} \mathrm{y}_{\mathrm{j}}
$$

The value of this output depends on the relative strengths of weighted input signals. The perceptron output can be expressed as:

$$
y(n)=f\left[w^{T}(n) x(n)+b\right]
$$

where, $w(n)=\left[w_{1}(n)\right.$ $\left.\mathrm{w}_{\mathrm{N}}(\mathrm{n})\right]$ is the adaptive weight vector $\mathrm{x}(\mathrm{n})=\left[\mathrm{x}_{1}(\mathrm{n}) \ldots \ldots \ldots \ldots \mathrm{x}_{\mathrm{N}}(\mathrm{n})\right] \mathrm{T}$ is the input signal vector, and $\mathrm{b}$ is the bias term. The most commonly used activation functions are sigmoid \& hard limiter. The perceptron weights are updated according to:

$$
\mathrm{w}(\mathrm{n}+1)=\mathrm{w}(\mathrm{n})+\eta[\mathrm{d}(\mathrm{n})-\mathrm{y}(\mathrm{n})] \mathrm{x}(\mathrm{n})
$$

where, $\eta$ denotes the learning rate parameter less than 1 and $\mathrm{d}(\mathrm{n})$ denotes the desired output or target.

\section{Limitation of Single Layer Perceptron}

The single layer perceptron model does not work in case of $\mathrm{X}-\mathrm{OR}$, we need more complex network that combine various simple networks together or can use different activation functions.

\section{B. Hopfield Neural Network}

Hopfield neural network is a form of recurrent artificial neural network which was invented by John Hopfield. In this network the output of each unit is fed as input to all other units except itself. The Hopfield network is used for pattern storage task. The main motive in a pattern storage task is to store a given set of patterns, and recall the corresponding or closest match pattern when an approximate version of the corresponding pattern is given to the network [8].

For this purpose, the features and their spatial relationship in the pattern need to be stored. The recall of a pattern should also take place when due to noise or distortion or due to variation of pattern generating process the feature and their spatial relations are slightly disturbed [8].

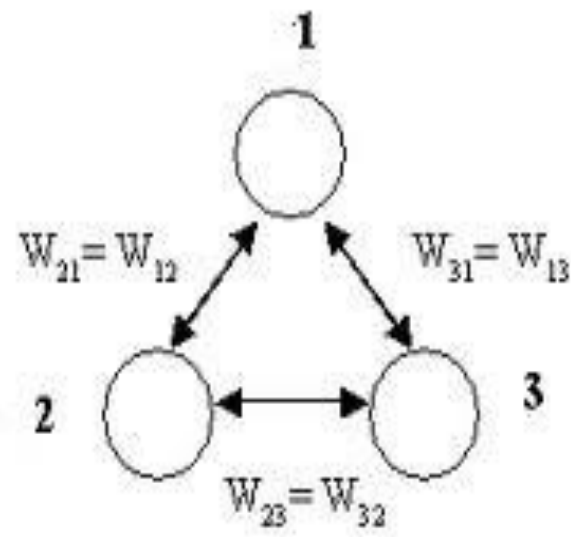

Fig 3: Simple Hopfield network with 3 nodes

The Hopfield algorithm for storing and recalling a pattern is given below [8]:

Let us consider a network consisting of $\mathrm{N}$ fully connected units with each unit having a hard-limiting bipolar threshold output function. Let $a_{1}, 1=1,2 \ldots$ be the vectors that we want to store. The vector $\left\{a_{1}\right\}$ are assumed to have bipolar components, i.e., $a_{1 i}= \pm 1, i=1,2, \ldots . . N$.

1. Initialize the network output with the given unknown input pattern a

$$
\begin{aligned}
& \mathrm{s}_{\mathrm{i}}(0)=\mathrm{a}_{\mathrm{i}}, \\
& \text { for } \mathrm{i}=1,2, \ldots \ldots . \mathrm{N} \\
& \text { where }
\end{aligned}
$$

$\mathrm{S}_{\mathrm{i}}(0)$ is the output of unit $\mathrm{i}$ at time $\mathrm{t}=0$ 
2. Assign the connection weights

$$
\begin{array}{rlrl}
\mathrm{w}_{\mathrm{ij}}=\sum_{\mathrm{i}=1}^{\mathrm{L}} \mathrm{a}_{\mathrm{li}} \mathrm{a}_{\mathrm{lj}} & \text { for } \mathrm{i} \neq \mathrm{j} \\
& =0, & \text { for } \mathrm{i}=\mathrm{j}, 1 \leq \mathrm{i}, \mathrm{j} \leq \mathrm{N}
\end{array}
$$

3. Iterate until convergence

$$
\mathrm{s}_{\mathrm{i}}(\mathrm{t}+1)=\operatorname{sgn} \sum_{j=1}^{\mathrm{N}} \mathrm{w}_{\mathrm{ij}} \mathrm{s}_{\mathrm{j}}(\mathrm{t}), \quad \text { for } \mathrm{i}=1,2,3 \ldots \ldots, \mathrm{N}
$$

This process is repeated till there is no change in the output with further iterations. The steady outputs of the units represent that this is the stored pattern that best matches the specified input.

\section{Limitations of Hopfield Neural Network:}

- $\quad$ Problem of False Energy Minima: There may be more energy minima or stable states in the network. Some of them correspond to desired patterns and other extra states correspond to false minima. The presence of extra stable state may result in recalling a pattern not in the set of desired patterns to be stored [8].

- Hard Problem: Pattern storage problems which cannot be represented by a feedback network of a given size are called as hard problems [8].

\section{Back-propagation Algorithm}

In Handwritten digit recognition system, a Backpropagation algorithm is used for training of the network.

Using back propagation, network weights are updated accurately so as to minimize errors [3].

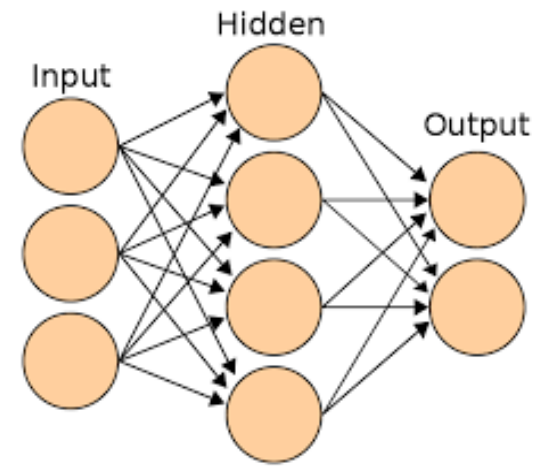

Fig 4: Neural Network

Back-propagation algorithm consists of two phases. First phase is the forward phase and the second phase is the backward phase. In the forward phase the activations move from the input layer to the output layer. In the second phase i.e. the backward phase the observed actual value and the requested nominal value in the output layer are propagated backwards so that the weights and bias values can be changed [5].

Back-propagation algorithm is described as follows [7]:

1) Initialize input layer which includes an input for bias. $\mathrm{I}_{\mathrm{i}}, \mathrm{W}_{\mathrm{i}}, \mathrm{T}_{\mathrm{i}}, \mathrm{Y}_{\mathrm{i}}$ Where, $\mathrm{I}_{\mathrm{i}}=$ input neurons, $\mathrm{W}_{\mathrm{i}}=$ random weights, $\mathrm{T}_{\mathrm{i}}=$ target values, $\mathrm{Y}_{\mathrm{i}}=$ output at each neuron.

2) Propagate activity forward through input layer to output layer.

$$
\mathrm{I}=>\mathrm{H}=>\mathrm{O}
$$

3) Calculate output at each neurons at each layer

$$
\mathrm{O}_{\mathrm{i}}=\Sigma \mathrm{I}_{\mathrm{i}} * \mathrm{~W}_{\mathrm{i}}
$$

4) Apply activation function to neurons and collect final output at each neuron

5) Calculate the error in the output layer

$$
\text { Error }=1 / 2\left(\mathrm{Y}_{\mathrm{i}}-\mathrm{T}_{\mathrm{i}}\right) 2
$$

Back propagate the error through layer

$$
\mathrm{dE} / \mathrm{dW}_{\mathrm{t}}=\mathrm{d}(\text { Error }) / \mathrm{dW}_{\mathrm{t}}
$$

Update the weights

$$
\Delta \mathrm{W}_{\mathrm{t}}=-\varepsilon\left(\mathrm{dE} / \mathrm{dW}_{\mathrm{t}}\right)+\alpha\left(\Delta \mathrm{W}_{\mathrm{t}-1}\right)
$$

Where, $\varepsilon=$ learning rate

$$
\alpha=\text { momentum }
$$

\section{CONCLUSION AND FUTURE SCOPE}

In this paper three approaches of neural network have been presented to recognize the handwritten digits. Among the all three approaches Back-propagation is the most successful in the recognition process. Back-propagation is fast and efficient as compared to the other two approaches and gives quick convergence on satisfactory local minima in case of error. It has a simple implementation and computing time is reduced if the weights chosen are small at the beginning.

In future we are expecting to explore the Back-propagation algorithm to make recognition of digits more fast and efficient and improve the overall performance.

\section{REFERENCES}

[1] Nasser Omer Sahel Ba-Karait and S.M. Shamsuddin, "Handwritten Digits Recognition using Particle Swarm Optimization", Second Asia International Conference on Modelling \& Stimulation.

[2] Bi Ran and Leong Tze Yuny, "Hand written digit recognition and its improvement", available at cs2306-machine learning.googlecode.com/svn/trunk/CS2306S.../a.pdf

[3] Ishani patel, Virag Jagtap and Ompriya Kale, "A Survey on Feature Extraction Methods for Handwritten Digits Recognition", International Journal of Computer Applications Volume 107 - No 12, December 2014

[4] Gaurav Kumar, Pradeep Kumar Bhatia, "Neural Network based Approach for Recognition of Text Images", International Journal of Computer Applications Volume 62- No.14, January 2013

[5] Malothu Nagu, N. Vijay Shankar and K.Annapurna, "A novel method for Handwritten Digit Recognition with Neural Networks", International Journal of Computer Science and Information Technologies, Vol. 2 (4), 2011.

[6] Jagyanseni Panda, Manaswinee M. Panda, Aryapriyanka Samal and Niva Das, "Odia Handwritten Digit Recognition Using Single Layer Perceptron", International Journal of Electronics and Communication Engineering and Technology(IJECET) Volume 5, Issue 4, April (2014)

[7] Viragkumar N. Jagtap and Shailendra K. Mishra,"Fast Efficient Artificial Neural Network for Handwritten Digit Recognition",International Journal of Computer Science and Information Technologies, Vol. 5, 2014.

[8] B. Yegnanarayana, "Artificial Neural Network", Eastern Economy Edition 